\title{
Fuzzy Logic based Odour Classification System in Electronic Nose
}

\author{
Jayeeta Sharma \\ Student, \\ Department of Electronics and \\ Communication Engineering, \\ Tezpur University,
}

\author{
Amlan Jyoti Bhagawati \\ Student, \\ Department of Electronics and \\ Communication Engineering, \\ Tezpur University,
}

\author{
Riku Chutia \\ Assistant professor \\ Department of Electronics and \\ Communication Engineering, \\ Tezpur University,
}

\begin{abstract}
In this paper, a Sugeno based fuzzy logic classifier for the classification of gases has been presented. The system employs an array of five gas sensors for sensing different gases. The sample space consists of eight gases. A database has been developed using experimentally collected data from the responses of the sensors. The classifier has been designed using the Fuzzy toolbox in MATLAB.
\end{abstract}

\section{Keywords}

Fuzzy Logic, Sugeno, Gas Sensor.

\section{INTRODUCTION}

In the 1960s, a graduate student at Columbia University, Lofti Zadeh proposed fuzzy logic [1]. Fuzzy logic has proved reliable in a number of industrial applications. One of the best known industrial fuzzy logic applications is the control system of the Sendai underground railway in Japan, utilized by Hitachi Company [2].

The development of gas sensors for the detection of gases has been carried out and has become a growing interest within the research community [3]. These sensors are widely used in applications in electronic nose [4].

An electronic nose refers to an instrument which consists of an array of gas sensors with partial selectivity and an appropriate pattern-recognition system, capable of recognizing simple or complex odours [5]. The electronic nose is mainly composed of two components namely the sensing system and the pattern recognition system. Each chemical vapor presented to the sensor array produces a pattern characteristic of the vapor. The database of the pattern characteristics is used to train the pattern recognition system. This training process is used to configure the recognition system to produce unique classifications of each chemical so that they can be correctly identified [6]. Mostly non- linear output signals are produced by the sensors and signals are varying to different sensors. Although sensors responses are dependent on the concentration of target gas, impact of working environment, such as temperature, humidity, power fluctuation can make the sensors output signal not accurate [7].

There are several methods which can be used to classify and detect the odour of various gases. Classical olfactometry method is used to detect the emission of gases. Odour classification by this method is based on the assessment by a selected group of people. The outcome of this method may not be accurate and the cost is also very high [8].

Classification, which refers to detection of gases by using sensors, is the best method, which gives more accuracy. These sensors are available at low cost and offer high sensitivity [9].

Various kinds of sensors are available such as chemical sensors, metal oxide sensors, conducting polymer sensors, quartz crystal microbalance sensors, etc. Each sensor has different sensing mechanism. Gas sensors are devices that translate the changes in the concentration of gaseous chemical species into electrical signals by changing the conductivity of the metal oxide layer [10].

In this paper, a sugeno based fuzzy inference system for the classification of gases at a constant temperature has been presented. The simulations are performed using fuzzy logic toolbox for eight different gases namely butanol, acetic acid, acetone, benzene, chloroform, di-chloromethane, ethyl-acetate and sulfuric acid.

\section{EXPERIMENTAL}

The work aims at the detection of samples of gases using an array of gas sensors. Pure samples of the gases of interest are prepared in a $10 \mathrm{~L}$ flask at known low concentration of approximately 300ppm [11]. The responses of a number of gas sensors to different gas samples are experimentally collected at a constant temperature. From the experimentally recorded data sets, an array of five sensors is selected. The responses of the sensors to the introduced sample of gas are measured as voltage variations using potentiometric circuit [12]. This experimentally collected voltage data set lie within a range of 0 to 2.5 volt. The response of the sensors is expressed in the form of histograms. Figure 1 and figure 2 show the histograms for Acetic Acid and Chloroform respectively.

\section{CLASSIFICATION}

The classification of the gases is done using fuzzy logic based approach. The member functions and rules of the fuzzy inference system are defined. The inputs applied to the five sensors are combined by AND operations. By applying the rules to the FIS, the outputs are obtained. A fixed parameter is assumed for a particular gas sample. The output obtained from the system is verified based on the closeness of the obtained 
output to the assumed fixed value. The block diagram of the fuzzy system for odor classification is shown in figure 3 .

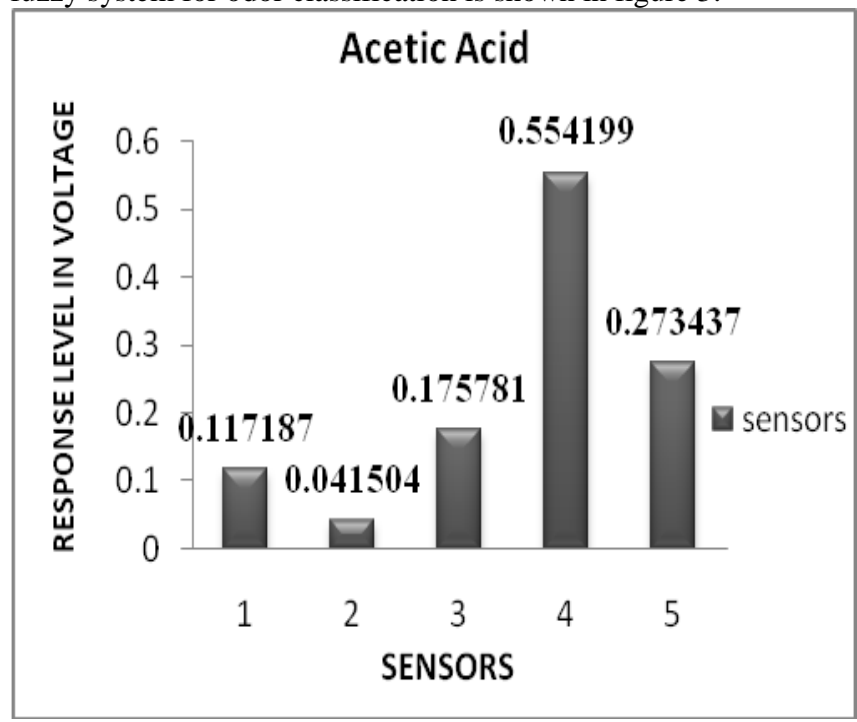

Fig 1: Histogram of Acetic Acid of Selected Sensors

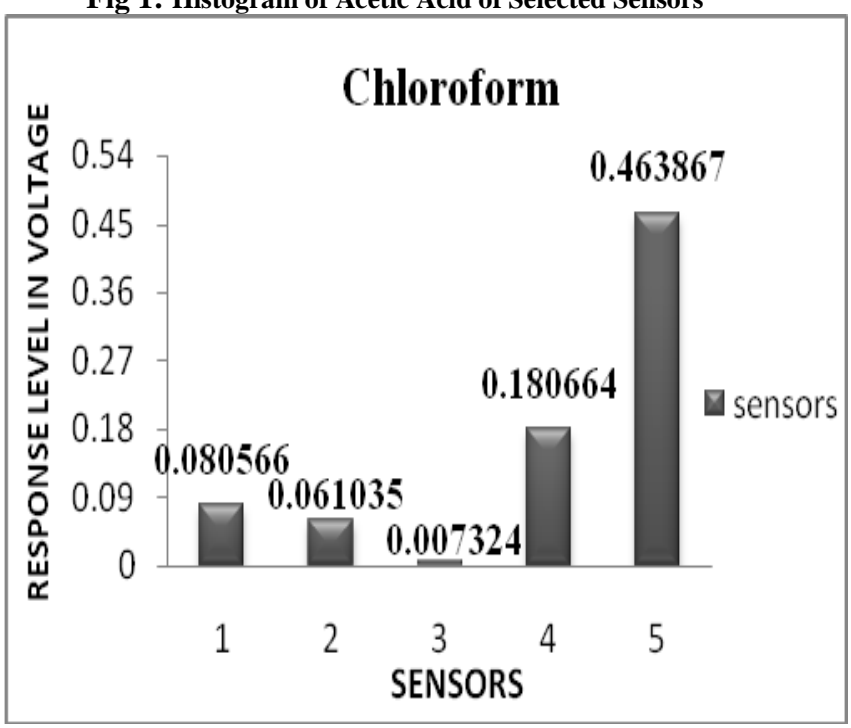

Fig 2: Histogram of Chloroform of Selected Sensors

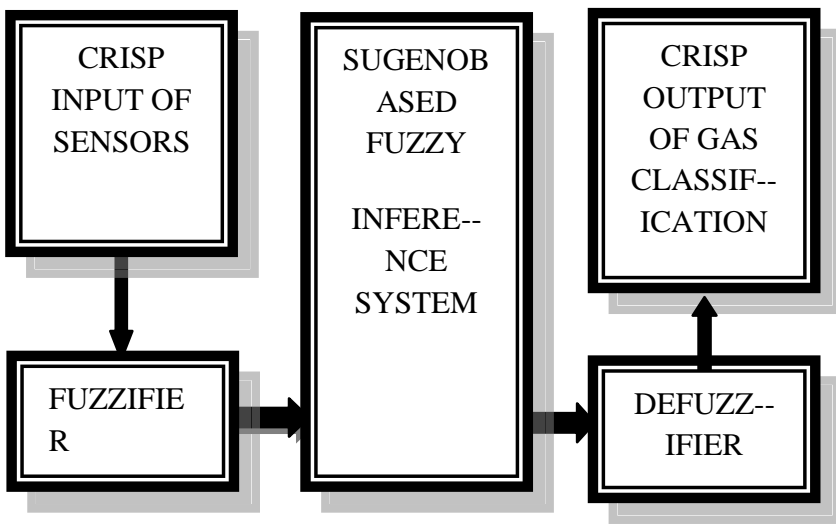

Fig. 3: Block Diagram of Sugeno Based Fuzzy System Used for Classification of Gases
The steps involved in the fuzzy logic classifier are described below:

\subsection{Fuzzification}

The first step in applying Sugeno based fuzzy logic algorithm is to fuzzify the inputs using membership functions. A membership function (MF) is a curve that defines how each input is mapped to a membership value (or degree of membership) between 0 and 1 [8]. The input values refer to the voltage levels given by the sensors. The inputs are fuzzified using nine triangular shaped member functions. The fuzzification of the input voltage levels is shown in figure 4 .

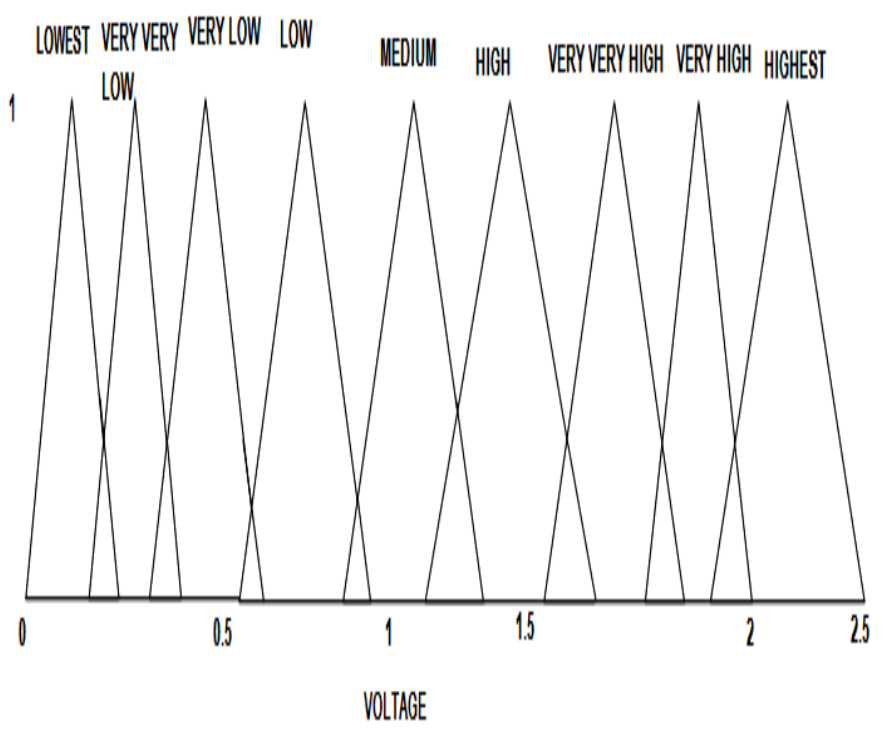

Fig. 4: Fuzzification of the Input Voltage Level

\subsection{Fuzzy Inference System}

The relationship between the input of the five sensors and the output gas samples is determined by the fuzzy rules in the inference system. A fuzzy control rule refers to a fuzzy conditional statement in which the antecedent is a condition in the application domain and the consequent is a control action for the system [13]. Some of the fuzzy rules are given as:

Rule 1: If sensor1 is very low AND sensor2 is medium AND sensor3 is medium AND sensor4 is highest AND sensor5 is low, then output is BUTANOL

Rule 2: If sensor1 is low AND sensor2 is medium AND sensor3 is medium AND sensor4 is highest AND sensor5 is low, then output is BUTANOL

Rule 3: If sensor1 is lowest AND sensor2 is lowest AND sensor3 is very very low AND sensor4 is very low AND sensor5 is very very low, then output is ACETIC ACID.

Rule 4: If sensor1 is lowest AND sensor2 is lowest AND sensor3 is very very low AND sensor4 is low AND sensor5 is very very low, then output is ACETIC ACID.

Figure 5 shows the FIS Diagram Depicting Inputs, Rule Base and Outputs. 


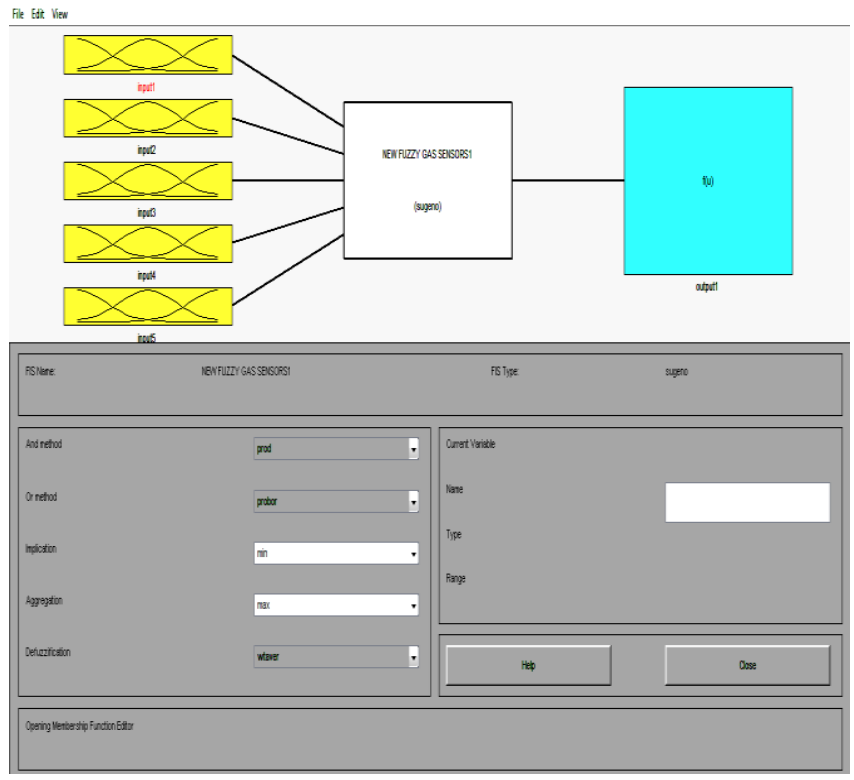

Fig. 5: FIS Diagram Depicting Inputs, Rule Base and Outputs.

\subsection{Defuzzification}

In the Sugeno FIS system, there is no output membership function at all. Instead the output is a crisp number computed by multiplying each input by a constant and then adding up the results. Using the Fuzzy toolbox, the crisp output values for the applied set of inputs are obtained. Figure 6 shows the experimental results for classification of the gases where voltage responses of five different sensors are 0.142, 0.09, $0.21,0.354$, and 0.3 respectively which gives a crisp output 2 that denotes Acetic acid.

\section{RESULTS AND DISCUSSIONS}

A set of voltage responses of the gas sensors to different gases was experimentally collected. Out of these, five sensors were selected for carrying out this project. Classification of gas was performed by sugeno based fuzzy logic system in MATLAB. The voltage responses given by different sensors were fuzzified using nine triangular shaped membership functions. A set of unique rules were defined for identification of the individual gases. A fixed parameter was assumed for a particular gas sample. The output obtained from the system was verified based on the closeness of the obtained output to the assumed fixed value. After developing the algorithm, tests were carried out at different data points over a range of constant temperature to find out the classification accuracy of the designed system. For each gas sample, the test was performed at ten different points. For eight gas samples, test was carried out for a total of 80 data points out of which correct results were obtained for 75 data points. Therefore, the gas classifier designed as stated above shows a classification accuracy of $93.75 \%$. When the classification of odours was investigated using back propagation neural network, the simulation using matlab function TRAINLM showed an efficiency of $100 \%$ [14]. In the experiment performed by Meegahapola et.al, for the same set of gases, fuzzy logic approach gives around $94 \%$ accuracy and it is close to $97 \%$ for the neural network based approach [8].

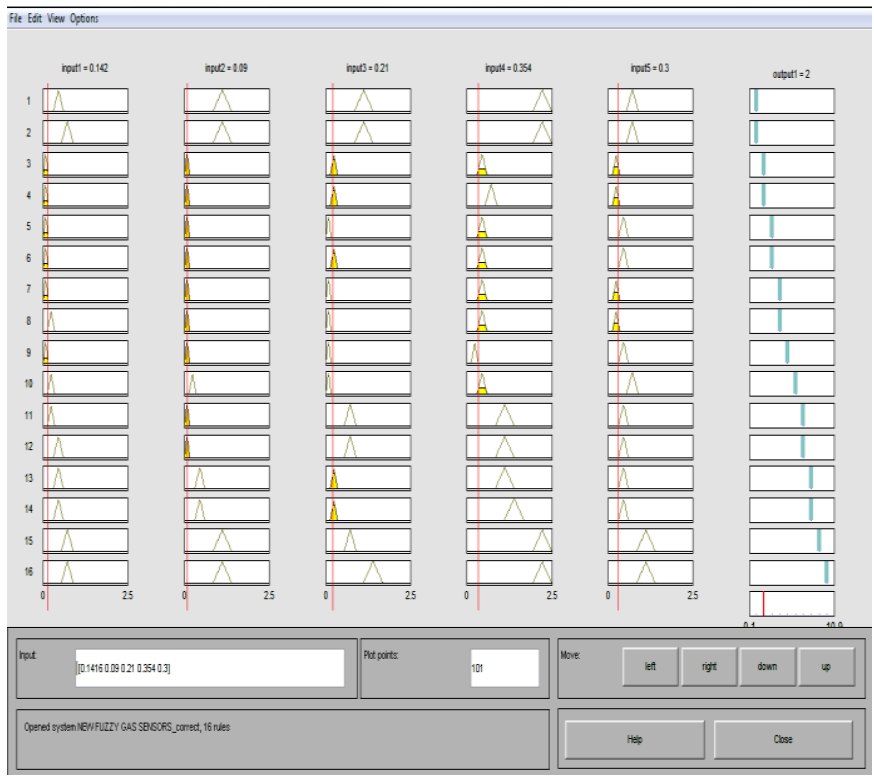

Fig. 6: Experimental Results for classification of Gas using Fuzzy logic in MATLAB

\section{CONCLUSION}

The main aim of the experiment was to classify different gases using Fuzzy logic approach. The experimental results showed that classification accuracy up to $93.75 \%$ can be obtained in this method. Therefore it can be concluded that in future, such an approach can be applied to other sample gases to check the accuracy of classification. Also by using different combinations of the gas sensor arrays, different results can be reported.

\section{ACKNOWLEDGMENTS}

We would like to thank the department of Electronics and Communication Engineering, Tezpur University for providing proper laboratory facilities for carrying out this project.

\section{REFERENCES}

[1] M. F. Abu Hassan, Y. Yusof, M.A. Azmi, and M.N. Mazli, "Fuzzy Logic Based Intelligent Control of RGB Colour Classification System for Undergraduate Artificial Intelligence Laboratory", Proceedings of the World Congress on Engineering 2012 Vol. II WCE 2012, July 4 - 6, 2012, London, U.K., ISBN: 978-988-19252-13 ISSN: 2078-0958 (Print); ISSN: 2078-0966 (Online).

[2] Kazuo Tanaka, "An Introduction to Fuzzy Logic for Practical Applications", Springer, 1996.

[3] N. Dutta, M Bhuyan, "Dynamic Response Based Odour Classification Using MOS Gas Sensor"s, 2011 Second International Conference on Emerging Applications of Information Technology, pp. 231-234, IEEE COMPUTER SOCIETY @2011 IEEE.

[4] R. Chutia and M. Bhuyan, "Online concentration independent feature dimension reduction of metal oxide gas sensor based E-Nose", IEEE-International Conference on Advances in Engineering, Science and Management (ICAESM -2012), pp. 247-250, March 30, 31, 2012. 
[5] Emmanuelle Schaller, Jacques O. Bosset and Felix Escher, "Electronic Noses' and Their Application to Food", Lebensm.-Wiss. u.-Technol., 31, pp. 305-316 (1998), Article No. fs980376, 0023-6438/98/040305 + 12 (01998 Academic Press.

[6] Paul E. Keller, Lars J. Kangas, Lars H. Liden, Sherif Hashem, Richard T. Kouzes, "ELECTRONIC NOSES AND THEIR APPLICATIONS", pp. 116-119 ISBN\# 07803-2639-3.

[7] Hua Fu, Tao Wang, Cui Yang, "Gas Monitoring System Based on the Multi-Sensor Information Fusion", The Ninth International Conference on Electronic Measurement \& Instruments, ICEMI'2009, 2-930-2-933, 978-1-4244-3864-8/09 @2009 IEEE.

[8] Lasantha Meegahapola, J.P.Karunadasa, Kasun Sandasiri, Damith Tharanga, Dammika Jayasekara, "Fuzzy Logic and Artificial Neural Network Approaches in Odor Detection", ICIA 2006, pp. 92-97,1-4244-05556/06@2006 IEEE.

[9] R. Chutia, M. Bhuyan, "Study of Temperature Modulated Tin Oxide Gas Sensor and Identification of Chemicals", CISP 2012|Proceedings|, pp. 181-184, 9781-4577-0720-9/12@2012 IEEE.

[10] Chambon, L., Germain, J. P., Pauly, A., Demarne, V., Grisel, A. 1999, "A metallic oxide gas sensor array for a selective detection of the $\mathrm{CO}$ and $\mathrm{NH}_{3}$ gases". Sensors and Actuators B 60, pp. 138-147.

[11] A.Uyanik and N.Tinkilic, "Preparing Accurate Standard Gas Mixtures of Volatile Substances at Low Concentration Levels", Chem. Educator 1999, 4, pp. 141-143@ 1999 Springer-Verlag, New York, Inc.

[12] Byeongdeok Yea, Ryosuke Konishi, Tomoyuki Osaki, Kazunori Sugahara, "The discrimination of many kinds of odor species using fuzzy reasoning and neural networks", Sensors and Actuators A 45 (1994), pp. 159165, 0924-4247/94/01 994 Elsevier Science S.A. All rights reserved SSDI 0924-4247/94)00831-2.

[13] Chuen Chien Lee, "Fuzzy Logic in Control Systems: Fuzzy Logic Controller-Part 1", IEEE TRANSACTIONS ON SYSTEMS, MAN AND CYBERNETICS, VOL. 20, No. 2, MARCH/ APRIL, 1990, pp. 404-418, 00189472/90/0300-0404@1990 IEEE.

[14] Ravi Kumar, R.R. Das, V.N. Mishra, R. Dwivedi, "A Fuzzy Logic Based Neural Network Classifier for Qualitative Classification of Odors/Gases", 2009 International Conference on Emerging Trends in Electronic and Photonic Devices \& Systems (ELECTRO-2009). 